\title{
Construction of compactly supported orthonormal symmetric and antisymmetric two-direction refinable functions and its corresponding wavelet with scale $M$
}

\author{
LI Wan-she ${ }^{a}$, YAO Quan-gang ${ }^{b}$ \\ College of Mathematics and Information Science, \\ Shannxi Normal University, Xi'an 710062, Shaanxi, China \\ aemail: liwsh@snnu.edu.cn, bemail:I
}

Key words: symmetric and antisymmetric; two-direction refinable functions; two-direction wavelet; compactly; orthonormal

\begin{abstract}
In this paper, the concepts and related conclusions of two-direction multiresolution analysis and two-direction refinable functions are introduced; two-direction wavelet with scale 2 is researched and is extended to orthonormal symmetric and antisymmetric two-direction wavelet with scale $\mathrm{M}$ and relevant properties are obtained. A condition that $L^{2}$ stable solution of two-direction refinable functions can create for MRA is given; finally construction of two-direction wavelet by two-direction compactly orthonormal refinable functions is discussed.
\end{abstract}

\section{Introduction}

Wavelet analysis theory originated in the $1970 \mathrm{~s}$, it is a rapid development of new fields. At present, it has been widely used in signal analysis, image processing, Quantum mechanics, theoretical physics; Military electronic countermeasures and weapons intelligent; Computer classification and recognition; Music and language of synthetic; Medical imaging and diagnosis; Seismic data processing; Large mechanical fault diagnosis, etc [1-4]. The different wavelet has been constructed according to the actual needs. Daubechies [5] have proved that in addition to haar wavelet beyond, for with orthogonal, symmetry and compact support with 2-band wavelet was not exist. In order to solve this problem, People have taken 2-band wavelet spread to multi- wavelet, multidimensional wavelets and made a deep research and gave the structure method. However, due to the Multiwavelets its limitations, there may be problems in the practical application. Literature [10-11] have detailed explore the multiwavelets balance problems.

Yang S Z [12-13] put forward the concept of two-direction refinable functions, and introduces the bidirectional orthogonal scaling function and wavelet function, and then got a few more practical theory and application results. Two-direction refinement equation is the promotion of refinement equation, So based on the bidirectional orthogonal refinement function can get good nature of the two-direction orthogonal wavelet. Xie C Z [14-15] researched the nature of the two-direction wavelet, and gives the structure two-direction wavelet method and example. This text combining with the two-direction wavelet, symmetric antisymmetric and promote literature 2-scale orthogonal symmetric wavelet to M-scale orthogonal symmetric and antisymmetric. Symmetric and antisymmetric two direction wavelet as a kind of new wavelet, it can be more widely used in more fields in real life.

\section{Orthonormal Two-Direction Refinable Function and Two-Direction Multi-Resolution Analysis}

Suppose $\phi \in L_{2}(R)$ is two-direction refinable functions, satisfies

$$
\phi(x)=\sum_{k \in Z} p_{k}^{+} \phi(m x-k)+p_{k}^{-} \phi(k-m x),
$$

In this formula $\left\{p_{k}^{+}\right\}$is $m(m \geq 2)$ forward scale sequence, $\left\{p_{k}^{+}\right\}$is $m$ negative scale sequence. 
For (1) on both sides simultaneously Fourier transform has:

$$
\hat{\phi}(\omega)=P^{+}\left(e^{-i \omega / m}\right) \hat{\phi}(\omega / m)+P^{-}\left(e^{-i \omega / m}\right) \hat{\phi}(\omega / m),
$$

where $p^{+}(z)=(1 / m) \sum_{k \in Z} p_{k}^{+} z^{k}$, is called forward two scale sequence symbol, and $p^{-}(z)=(1 / m) \sum_{k \in Z} p_{k}^{-} z^{k}$ is called negative two scale sequence symbol.

Definition 1 If there are two positive constant $A$ and B, such that

$$
A \sum_{k \in Z} c_{k} c_{k}^{*} \leq\left\|\sum_{k \in Z} c_{1}^{k} \phi(x-k)+\sum_{k \in Z} c_{2}^{k} \phi(k-x)\right\|_{2}^{2} \leq B \sum_{k \in Z} c_{k} c_{k}^{*}
$$

for any sequences $\left\{c_{k}\right\}_{k \in Z}=\left\{\left[c_{1}^{k}, c_{2}^{k}\right]\right\}_{k \in Z} \in I^{2}\left(Z^{2}\right)$. In order to study the solution existence of (1), Though the deformation of (1), we get that

$$
\phi(-x)=\sum_{k \in Z} p_{k}^{+} \phi(-m x-k)+p_{k}^{-} \phi(k+m x)_{o}
$$

Let

$$
\Phi(x)=\left[\begin{array}{l}
\phi(x) \\
\phi(-x)
\end{array}\right]=\sum_{k \in Z}\left[\begin{array}{cc}
p_{k}^{+} & p_{k}^{-} \\
p_{-k}^{-} & p_{-k}^{+}
\end{array}\right]\left[\begin{array}{c}
\phi(m x-k) \\
\phi(k-m x)
\end{array}\right]=\sum_{k \in Z}\left[\begin{array}{cc}
p_{k}^{+} & p_{k}^{-} \\
p_{-k}^{-} & p_{-k}^{+}
\end{array}\right] \Phi(m x-k)
$$

Now, we introduce autocorrelation matrix symbol of the two-direction refinable function $\phi(x)$ :

$$
\Omega(\omega)=\sum_{k \in Z}\left[\begin{array}{cc}
<\phi(x), \phi(x-k)> & <\phi(x), \phi(k-x)> \\
<\phi(-x), \phi(x-k)> & <\phi(-x), \phi(k-x>
\end{array}\right] e^{-i k x}
$$

If 1 is a characteristic value of a matrix or operator, and module of other characteristic value is less than 1, the matrix or operator satisfies the condition $E$.

Define sequences of subspaces $\left\{V_{j}\right\} \subset L^{2}(R)$, where

$$
V_{j}=\operatorname{close}_{L^{2}(R)}<m^{j / 2} \phi\left(m^{j} x-k\right), m^{j / 2} \phi\left(l-m^{j} x\right)>
$$

and, then can generate a two-direction multiresolution analysis if and only if of (7) satisfy the following conditions:
(i) $\ldots \subset V_{-1} \subset V_{0} \subset V_{1} \subset \ldots$;
(ii) close $\left._{L^{2}(R)}\left\{\cup_{j \in Z} V_{j}\right)\right\}=L^{2}(R)$;
(iii) $\underset{j \in Z}{\cap}\left(V_{j}\right)=\{0\}$;
(iv) $f(x) \in V_{j}$ if and only if $f(m x) \in V_{j}$;

(v) $\{\phi(x-k), \phi(n-x), k, n \in Z\}$ be a Riesz base of $V_{0}$.

Definition 2 Let $\phi(x)$ is compact support bidirectional refinable function, we call that $\phi(x)$ is two-direction orthogonal refinable function if

$$
<\phi(x), \phi(x-k)>=\delta_{0, k}, \quad<\phi(x), \phi(n-x)>=0, \quad k, n \in Z,
$$

hold. Multi-resolution analysis which a two-direction orthogonal refinable function $\phi(x)$ generates is called two-direction orthogonal multiresolution analysis.

Definition 3 If the function $g(x)$ is symmetric (antyisymmetric) about $b$, then $g(b+x)=g(b-x)(g(b+x)=-g(b-x))$ holds for all .

By the above definition, For two-direction wavelet functions and two-direction refinable functions

$$
\psi(x)=\sum_{k \in Z} q_{k}^{+} \phi(m x-k)+\sum_{k \in Z} q_{k}^{-} \phi(k-m x), \phi(x)=\sum_{k \in Z} p_{k}^{+} \phi(m x-k)+\sum_{k \in Z} p_{k}^{-} \phi(k-m x) .
$$

They are symmetric or antyisymmetric if and only if $\phi(m x-k)$ and $\phi(k-m x)$ are symmetric or antyisymmetric.

Theorem 1 The orthogonal compactly supported two-direction refinable function $\phi(x)$ is antisymmetric about point $a$, and such that $\phi(x)=\sum_{k \in Z} p_{k}^{+} \phi(m x-k)+\sum_{k \in Z} p_{k}^{-} \phi(k-m x)$.

Then we have $p_{k}^{+}=-p_{m a-k}^{+}, p_{k}^{-}=-p_{m a-k}^{-}$. 
Proof. Since $\phi(x)$ is antisymmetric about point $a$, we know that $\phi(x+a)=-\phi(a-x)$, i.e, $\phi(x+a)=\sum_{k \in Z} p_{k}^{+} \phi(m x+m a-k)+\sum_{k \in Z} p_{k}^{-} \phi(k-m x-m a)$.

$$
\phi(a-x)=\sum_{k \in Z} p_{k}^{+} \phi(m a-m a-k)+\sum_{k \in Z} p_{k}^{-} \phi(k-m a+m x) .
$$

Since $\phi(m x-k)$ and $\phi(k-m x)$ are both antisymmetric, combined with Lemma $4^{[16]}$, we easily that get that $p_{k}^{+}=-p_{m a-k}^{+}, p_{k}^{-}=-p_{m a-k}^{-}$,

In the same way, For symmetrical and refinable function $\phi(x)$, we have $p_{k}^{+}=p_{m a-k}^{+}, p_{k}^{-}=p_{m a-k}^{-}$. Analog Theorem 2, we can get to meet the two-direction wavelet function $\psi(x)=\sum_{k \in Z} q_{k}^{+} \phi(m x-k)+\sum_{k \in Z} q_{k}^{-} \phi(k-m x)$. Then $\psi(x)$ is symmetric or antisymmetric about point $a$,we have

$$
q_{k}^{+}=q_{m a-k}^{+}, q_{k}^{-}=q_{m a-k}^{-}, q_{k}^{-}=q_{m a-k}^{-} ; \text {or } q_{k}^{+}=-q_{m a-k}^{+}, q_{k}^{-}=-q_{m a-k}^{-} .
$$

Theorm 2 Let $\phi(x)$ is a orthogonal two-direction and refinable function compactly supported symmetric and antisymmetric ,and such that

$$
\phi(x)=\sum_{k=0}^{n} p_{k}^{+} \phi(m x-k)+\sum_{k=0}^{n} p_{k}^{-} \phi(k-m x)
$$

Define $\Phi(x)=(\phi(m x), \phi(m x-1), \ldots, \phi(m x-(m-1)))^{T}$, and satisfies the conditions of the Theorem 1 , then $\Phi(x)$ is a orthogonal Compactly supported two-direction refinable function, and the support set $\Phi(x)$ is the subset of

\section{Proof:}

$$
\left[-\frac{n}{(m-1) m}, \frac{n}{(m-1) m}+\frac{m-1}{m}\right]
$$

$$
\begin{aligned}
& <\Phi(x), \Phi(x-n)>=\int_{R} \Phi(x) \overline{\Phi(x-n) d x} . \\
& =\int_{R}\left[\phi(m x), \ldots, \phi(m x-(m-1)]^{T}\left[\phi(m x-m n), \ldots, \phi(m x-m n-(m-1)] d x=\delta_{0, n} I .\right.\right.
\end{aligned}
$$

That completes Orthogonality.

Symmetry Since every $\phi(m x), \ldots, \phi(m x-(m-1))$ is symmetric or antisymmetric, we get easily that $\Phi(x)$ is symmetric or antisymmetric.

Compacted support By reference [13], we know that the support set of $\phi(x)$ is a subset of $\left[-\frac{n}{m-1}, \frac{n}{m-1}\right]$, so the support set of $\phi(m x)$ is $\left[-\frac{n}{(m-1) m}, \frac{n}{(m-1) m}\right]$, and the support set of $\phi(m x-(m-1))$ is $\left[-\frac{n}{(m-1) m}+\frac{m-1}{m}, \frac{n}{(m-1) m}+\frac{m-1}{m}\right]$. Therefore, the support interval of $\Phi(x)$ is the subset of $\left[-\frac{n}{(m-1) m}, \frac{n}{(m-1) m}+\frac{m-1}{m}\right]$.

According to Theorem 2 , we can similarly construct two-direction orthogonal symmetric and antisymmetric compactly supported wavelet functions.

\section{Conclusion}

In this paper, two-direction and refinable function and two-direction multi-resolution analysis

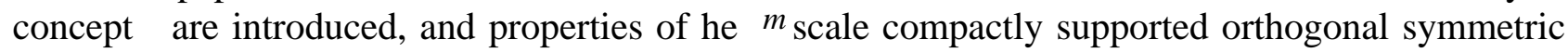
and antisymmetric two-direction wave are studied. In addition, we get general methods of construction of the compactly supported orthogonal symmetric and antisymmetric two-direction and refinable function corresponding bidirectional wavelet As for concrete construction methods and actual applications remain to be further research. 


\section{References}

[1] Xia T, Jiang Q T. Optimal multifilter banks: design, related symmetric extension transform and application to image compression . IEEE Transactions Signal Process, 1999, 47(7): 1878-1890.

[2] Tan H H, Shen L X, Than J Y. New biorthogonal multiwavelets for image compression [J]. IEEE Transform Signal Process, 1999, 79(1):45-65.

[3] Cheng Lizhi, Wang Hongxia, Wavelet theory and application. Beijing: Science press:2004.

[4] Telesca L, Lapenna V, Alexis V. Multiresolution wavelet analysis of earthquakes Chao Solutions \& Fractals, 2004, 22(3):741-748.

[5] I. Daubechies Ten Lectures on Wavelets . Beijing: National defence industry press.

[6] Chui C K, Lian J A. Compactly supported symmetric and antisymmetric orthonormal wavelet with scale 3 . Applied and Computational Harmonic Analysis, 1995, 2(1):21-51.

[7] Huang Yongdong, Cheng Zhengxing, Sun Lei. Structure symmetric and antisymmetric M with political filter new method. Journal of Engineering Mathematics,2005,22(5):781-786.

[8] Quan Hongyue, Wang Guoqiu. A kind of symmetric orthogonal multiwavelet structure [J]. Shaanxi normal university journal, 2008,31(4):682-691.

[9] Li Wanshe, Hao Wei, Meng Shaoting. a Scale wavelet orthogonal two-way Mallat algorithm . Shaanxi Normal University Journal(Natural Science), 2010,38(3): 1-5.

[10] Cui Lihong, Chengzhengxing. Many small wave and balanced multiwavelet theory and design . Journal of Engineering Mathematics, 2001,18(5): 105-116

[11] Cai Jianhong, Tao Feng. Multivariate wavelet filter matrix expansion. Acta Mathematica Scientia, 2009,29 (2) : 449-455

[12] Yang Shouzhi. Biorthogonal two-direction refinable function and two-direction wavelet . Journal of Applied Mathematics and Computing, 2006, 49(1):86-97

[13] Yang Shouzhi, Li Youfa. Two-direction refinable functions and two-direction wavelets with high approximation order and regularity . Sci China: A, 2007, 50(12): 1687 -1704.

[14] Xie Changzen, Yang Shouzhi. Orthogonal two-direction multiscaling functions . Frontiers of Mathematics in China, 2006, (4):604-611.

[15] Xie C Z. Construction of biorthogonal dilation factor m . Computers And Mathematics With Applications, 2008, 56:1854-1861.

[16] Li wanshe, Luo Lisuo, Li qiao. Orthogonal symmetric compactly supported two-way wavelet structure. Journal of Shantou University: Natural Science, 2011,26,(1):1-7. 